\title{
Quantitative tomography with subsurface scanning ultrasound resonance force microscopy
}

\section{Maarten H. van Es, Laurent Fillinger, Hamed Sadeghian}

Maarten H. van Es, Laurent Fillinger, Hamed Sadeghian, "Quantitative tomography with subsurface scanning ultrasound resonance force microscopy," Proc. SPIE 10959, Metrology, Inspection, and Process Control for Microlithography XXXIII, 109590M (2 April 2019); doi: 10.1117/12.2515048 


\title{
Quantitative tomography with Subsurface Scanning Ultrasound Resonance Force Microscopy
}

\author{
Maarten H. van $\mathrm{Es}^{\mathrm{a}}$, Laurent Fillinger ${ }^{\mathrm{a}}$, and Hamed Sadeghian ${ }^{\mathrm{a}}$ \\ ${ }^{a}$ NOMI, Netherlands Organization for Applied Scientific Research (TNO), Delft, The \\ Netherlands
}

\begin{abstract}
Extracting quantitative information about dimensions and material properties of buried structures is continuing to be an important but difficult task in metrology. Examples of questions asking for this capability include critical dimension metrology of fins such as the profile (bottom width, top width, height) or the presence and extent of voids. In recent years TNO has demonstrated the concept of using Atomic Force Microscopy (AFM) in combination with ultrasound to image buried structures based on their (visco-)elasticity in a technique called Subsurface Scanning Ultrasound Resonance Force Microscopy (SSURFM). We have successfully imaged structures less than $10 \mathrm{~nm}$ wide, as well as structures buried up to a micrometer deep. However, extracting quantitative information from this data is not trivial, as the induced stress field in the sample depends on many parameters in a non-linear way: experimental parameters such as applied force, tip size and tip shape, and geometry and material properties of the buried structures themselves. Therefore, measurements based on this technique have a point spread function which varies in a complicated way with the sample properties that need to be measured. However, a solid understanding of the physics and mechanics involved, and the modeling of the expected structures and their response to externally applied stress, enable quantitative measurements. We specifically show our progress on characterizing a sample comprising fins from a $7 \mathrm{~nm}$ node manufacturing test run.
\end{abstract}

Keywords: Buried structures, Critical Dimensions, Quantitative, Imaging

\section{INTRODUCTION}

Increasing geometrical complexity and ever smaller typical length scales in modern semiconductor design place ever higher demands on metrology. Not only do features get smaller, they also need to be characterized according to more degrees of freedom. In practice this means that more and more device features are difficult to assess from the top of the structures using traditional metrology tools based on electron beams or light. Examples include many critical dimensions of fin structures including height, bottom width, sidewall angle and sidewall roughness. For next generation structures, such as Gate All Around Nanowires, these issues will only be exacerbated. Figure 1 shows the fin design studied in this work in more detail, as well as experimental data on these fins from Scanning Subsurface Ultrasonic Resonance Force Microscopy (SSURFM) ${ }^{1}$ and (destructive) cross-sectional SEM.

Scanning Electron Microscopy (SEM) has been shown to be sensitive to at least some of those parameters for fins, ${ }^{2}$ but it has to rely heavily on the modeling of the trajectories of secondary electrons to relate measured signals to CD's. ${ }^{3}$ It is not clear if the measurements can be rich enough in information to unambiguously resolve all parameters describing a finfet. ${ }^{4}$ In addition, high resolution SEM is prone to damage materials due to the high energies involved. ${ }^{5}$ This is increasingly a problem as maintaining the material integrity becomes critical at smaller length scales.

Scanning Probe Microscopy (SPM) has sufficient resolution to resolve all relevant parameters ${ }^{6}$ of finFET and next generation structures without damaging them. ${ }^{7}$ However with increasingly thin and deep gaps between features and more and more complex geometries, the shape and rigidity of tips are fundamentally limiting the possibilities to employ SPM for characterizing fins using surface profiling. In recent years we have investigated

Further author information: (Send correspondence to M.H. van Es)

M.H. van Es: E-mail: maarten.vanes@tno.nl, Telephone: 0031888665685

Metrology, Inspection, and Process Control for Microlithography XXXIII, edited by

Vladimir A. Ukraintsev, Ofer Adan, Proc. of SPIE Vol. 10959, 109590M @ 2019

SPIE $\cdot$ CCC code: $0277-786 \mathrm{X} / 19 / \$ 18 \cdot$ doi: $10.1117 / 12.2515048$

Proc. of SPIE Vol. 10959 109590M-1 

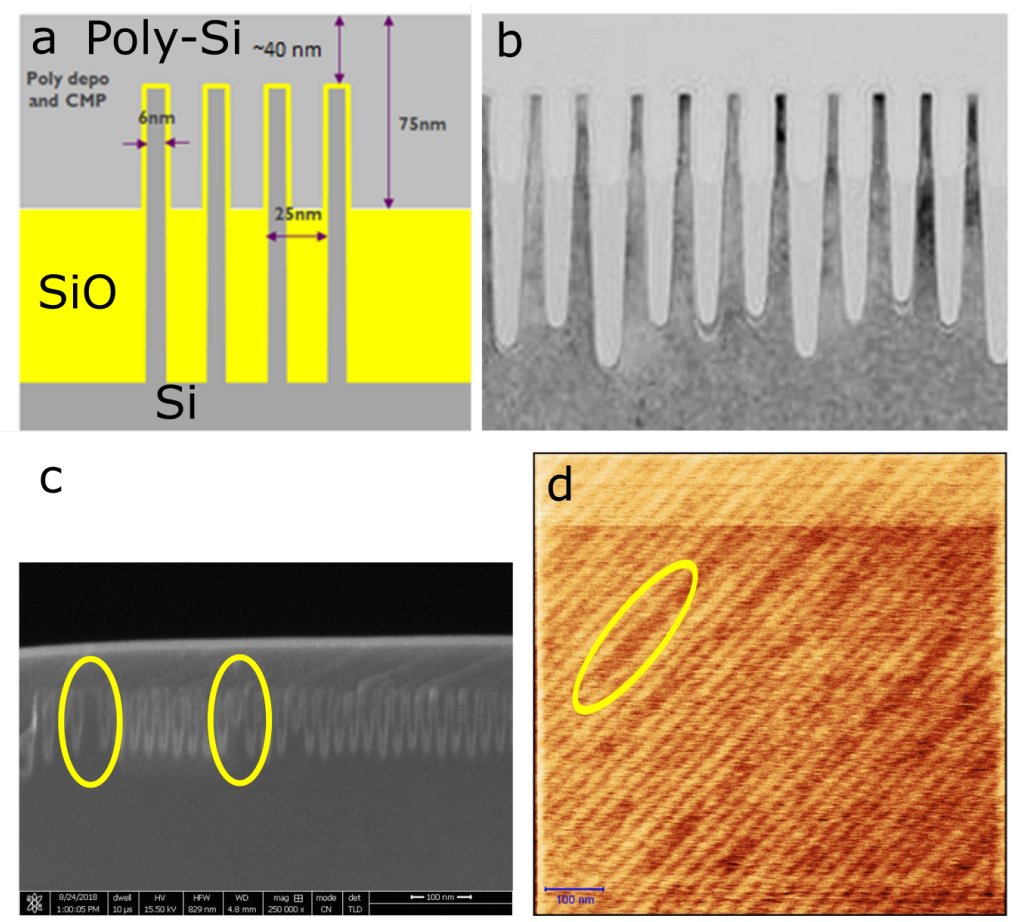

Figure 1. Sketch of fin design and images of the realized structure. This sample is from an early production run at IMEC where the production process was not yet fully optimized. (a) Design of fins; (b) cross-sectional SEM image of fins (courtesy of IMEC). The quadruple patterning process clearly has an impact on fin height and shape; (c) cross-sectional SEM image showing production faults in this early production sample, as indicated by the yellow circles; (d) SSURFM image of fins, the colour scale indicates here the amplitude of the SSURFM signal on an arbitrary scale. Clearly visible in this picture are defective areas which correspond (most likely) to the fin collapse faults shown also in (c).

and optimized the performance of subsurface imaging of structures based on their (visco-)elastic properties using SPM. ${ }^{1,8}$ Once the gaps between the structures have been filled with other materials, this technique, in principle, allows extracting all kinds of geometrical and material properties from the measured data. Thus it enables characterizing structures in 3 dimensions. In this paper, we take a closer look on the contrast mechanism of SSURFM highlighting how quantitative information can be extracted from the measurements. We also present an analysis of defect characterization using SSURFM.

\section{QUANTIFICATION OF S+SURFM}

The cantilever in SPM is sensitive to the mechanical properties of the sample under investigation. In particular, the resonance frequency of the cantilever plus tip in contact with the sample is given by properties of the cantilever such as geometry and material properties, and by the boundary conditions acting on the tip while it interacts with the sample. It is typically modeled using the classic Euler-Bernoulli equation for the motion of beams: $\frac{\partial^{2}}{\partial x^{2}}\left(E I \frac{\partial^{2} w}{\partial x^{2}}\right)=-\mu \frac{\partial^{2} w}{\partial t^{2}}+q$ with $w$ the cantilever displacement, $x$ the location along the cantilever, $\mu$ the mass per unit length, $E$ the Young's modulus of the beam material, $I$ the second moment of area of the beam's cross-section and $q$ the load distribution on the cantilever. The boundary conditions near the tip are determined by the relations between forces and deformations acting in the tip-sample contact; the other boundary conditions are determined by the attachment of the cantilever to the support chip. Buried structures alter these relation as can be appreciated by looking at the distribution of stress in the sample (see figure 2a,b for an example). Thus, recording the (eigen-)motion of the cantilever allows reconstructing the boundary conditions and through them the geometry and properties of buried structures. It may be necessary to reconstruct the boundary conditions as a function of e.g. tip position and applied force to gather enough information to reconstruct the buried features. In this paper we only consider the forces acting perpendicular to the sample surface as we restrict the applied force in this direction, ensuring this is the dominant direction in our experiments. It should be kept in mind 
that forces acting along the sample surface may help provide additional information by deforming the sample in different ways.

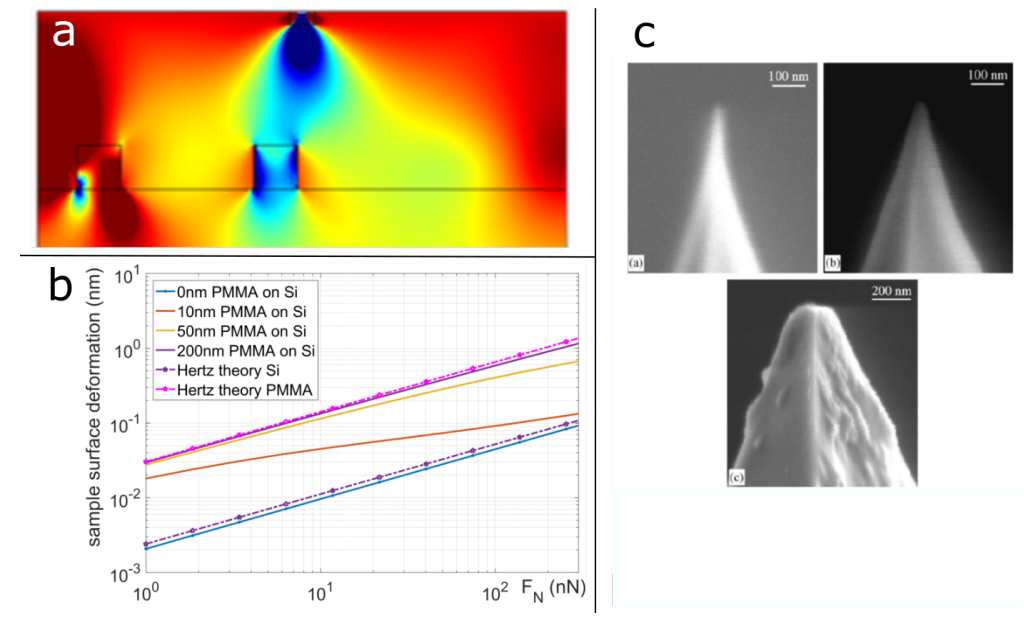

Figure 2. Several issues make interpretation of SSURFM data not so straightforward. (a) The distribution of stress in a sample due to a locally applied strain with the presence of buried structures. It is obvious that the buried structures influence the distribution of stress; it is challenging to figure out how this affects the tip-sample interaction; (b) Simulations can tell us the amount of deformation in the contact as a function of applied force, in this case on a sample consisting of 2 simple unstructured layers. (c) Another parameter which needs careful attention to quantitatively match experiments and simulations is the tip shape and diameter. Depicted here are images of a few new, unused tips of the same type. They differ quite significantly in diameter, which is one of the most important parameters in contact mechanics models.

Examining the boundary conditions in the tip-sample contact in more detail, the force applied by the tip on the sample surface causes the sample to deform. The induced stress-field has a complex distribution even for flat, homogeneous and isotropic samples. For such a homogeneous and isotropic sample and without adhesion forces acting between tip and sample, the force-indentation relationship has been derived and even solved analytically for several shapes of the indenter. ${ }^{9}$ From these results we already see that the shape of the tip has a significant influence on the force-indentation relationship and thus on the boundary conditions. However, the tip shape is not very well controlled for AFM tips as illustrated in figure 2c. This is a major experimental difficulty to adress to obtain quantitative information from SPM measurements. It can be solved either by carefully characterizing the tip shape on special purpose tip calibration samples ${ }^{10,11}$ or by performing calibration indentation measurements on a known sample. ${ }^{12}$ Rabe et al. has shown ${ }^{12}$ that layer thickness and material properties may be extracted from SSURFM meausrements in this way. Spatially mapping the response at high resolution will enable the measurement of the extent of buried plateaus at different depths, allowing the extraction of quantitative values for the size of those structures.

Additionally, direct high resolution imaging of fins allows quantification and classification of fins and their potential defects. Figure 3a shows an area we analyzed for the presence of defects. Prior to the defect detection, the following preprocessing is applied. A tilted rectangular area is selected, as indicated by the green rectangle in figure 3a. The corresponding portion of the image is flattened by removing the linear trend (average plane). We notice that, in absence of defects, the preprocessed image is characterized, across the trenches, by a succession of portions of high values, separated by relatively narrower portions of low values, whereas along the trenches the image is rather uniform. The presence of defects breaks this uniformity along the trenches, in particular in zones of low values, which are locally much wider than in defect-free area. The defect detection is then performed according to the following steps. First, a sequence of grayscale morphological erosion/dilatation ${ }^{13}$ is performed along and across the trenches. This sequence is designed to close the narrow gaps of low values between nondefective trenches, while leaving the larger gaps of low values corresponding to the defects. Figure 3c shows the resulting image from this step and clearly the defects remain visible as darker areas although the good trenches have been removed. The resulting image is then converted to a binary mask by thresholding. The threshold is automatically selected by analysis of the histogram of the values in the morphologically processed image, in order 

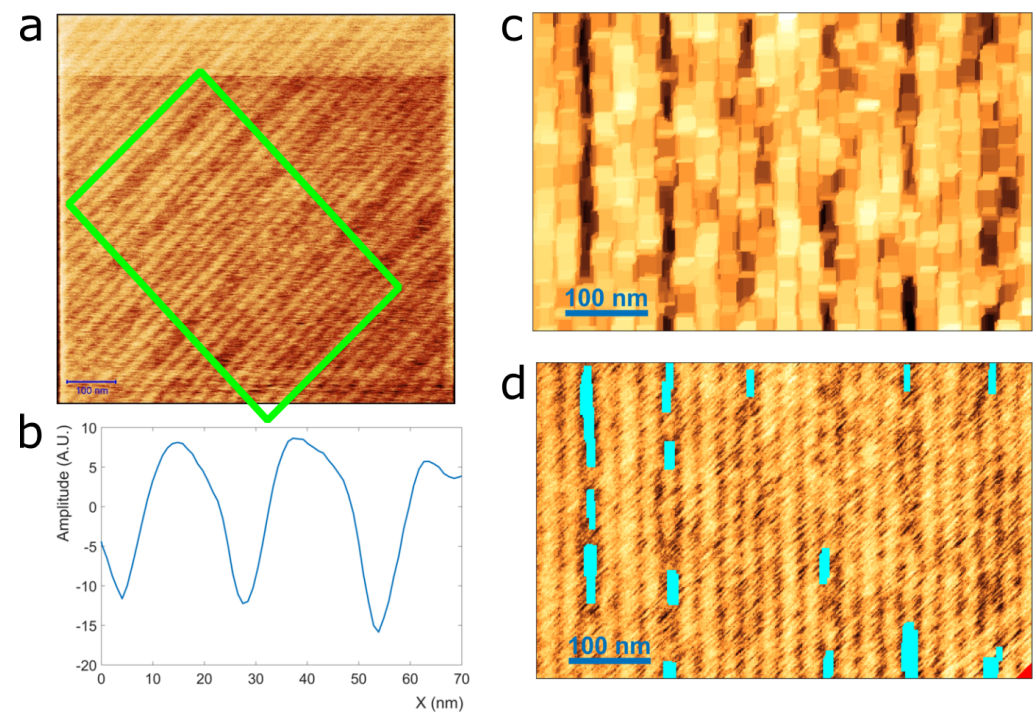

Figure 3. (a,b,c) Example of defect detection showing the processed image area (a), an intermediate processing step (b) and the detected defects (c). (d) Cross-section across the fins. The non-harmonic profile indicates that the information in this profile is not limited to the pitch.

to separate the relatively low values associated with defects, from the higher values corresponding to defect-free area. Another step of morphological treatment is applied to this binary image in order for the defect area to recover their apparent size in the pre-processed image. Finally, the blobs of connected defect pixels are identified, allowing the evaluation various quantitative metrics. Figure 3d highlights the defects identified in this manner. For each blob, the width, length and area is evaluated. For the image, the number of blobs, the average blob size, maximum blob length and the percentage of affected area are evaluated. Although some processing steps have been carried out manually, this processing can be implemented in a fully automatic way.

Our analysis identifies 14 individual defects in this image, of which 10 are fully within the field of view. All 14 defects occupy a surface area of $3.8 \%$ of the image; the 10 defects which are fully visible have a mean length of $53 \mathrm{~nm}$ and a maximum length of $124 \mathrm{~nm}$.

\section{CONCLUSIONS}

SSURFM can image small, nm-scale buried structures under arbitrary and optically opaque covering layers. This enables non-destructive evaluation of the quality of device features even in the latest semiconductor production nodes. Direct imaging of defects allows extracting quantitative information about the presence and extent of defects, which can support manufacturing process optimization. Further development of modeling and careful physical characterization will enable extraction of critical dimensions such as width and height of buried structures like fins or gate all around nanowire structures.

\section{ACKNOWLEDGMENTS}

This project has received funding from the Electronic Component Systems for European Leadership Joint Undertaking under grant agreement No 737479. This Joint Undertaking receives support from the European Union's Horizon 2020 research and innovation programme and Netherlands, France, Belgium, Germany, Czech Republic, Austria, Hungary, Israel

\section{REFERENCES}

[1] van Es, M. H., Mohtashami, A., Thijssen, R. M., Piras, D., van Neer, P. L., and Sadeghian, H., "Mapping buried nanostructures using subsurface ultrasonic resonance force microscopy," Ultramicroscopy 184, 209216 (jan 2018). 
[2] Bunday, B. D., Mukhtar, M., Quoi, K., Thiel, B., and Malloy, M., "Simulating massively parallel electron beam inspection for sub-20 nm defects," in [Metrology, Inspection, and Process Control for Microlithography XXIX], Cain, J. P. and Sanchez, M. I., eds., SPIE (mar 2015).

[3] Ma, Z. and Seiler, D., eds., [Metrology and Diagnostic Techniques for Nanoelectronics], CRC Press (nov 2016).

[4] Bunday, B., "HVM metrology challenges towards the 5nm node," in [Metrology, Inspection, and Process Control for Microlithography XXX], Sanchez, M. I. and Ukraintsev, V. A., eds., SPIE (mar 2016).

[5] Patterson, O. D., Hafer, R. F., Tang, X., and Lei, S.-C. C., "The benefits of high landing energy for e-beam inspection," IEEE Transactions on Semiconductor Manufacturing 29, 320-327 (nov 2016).

[6] Biemond, J. B., Herfst, R. W., Mashaghi, S., Dekker, B., Bijnagte, T., and Sadeghian, H., "Design and simulation of atomic force profiling of high aspect ratio samples using $2 \mathrm{~d}$ subresonant force spectroscopy," in [2018 IEEE/ASME International Conference on Advanced Intelligent Mechatronics (AIM)], IEEE (jul 2018).

[7] Keyvani, A., Sadeghian, H., Tamer, M. S., Goosen, J. F. L., and van Keulen, F., "Minimizing tip-sample forces and enhancing sensitivity in atomic force microscopy with dynamically compliant cantilevers," Journal of Applied Physics 121, 244505 (jun 2017).

[8] van Es, M. H., Mohtashami, A., van Neer, P. L., and Sadeghian, H., "Sounding out buried nanostructures using subsurface ultrasonic resonance force microscopy," MRS Advances 3(11), 603-608 (2018).

[9] Sneddon, I. N., "The relation between load and penetration in the axisymmetric boussinesq problem for a punch of arbitrary profile," International Journal of Engineering Science 3, 47-57 (may 1965).

[10] Ukraintsev, V., "Review of reference metrology for nanotechnology: significance, challenges, and solutions," Journal of Micro/Nanolithography, MEMS, and MOEMS 11, 011010 (feb 2012).

[11] Huebner, U., Morgenroth, W., Meyer, H., Sulzbach, T., Brendel, B., and Mirande, W., "Downwards to metrology in nanoscale: determination of the AFM tip shape with well-known sharp-edged calibration structures," Applied Physics A: Materials Science 63 Processing 76, 913-917 (apr 2003).

[12] Rabe, U., Kopycinska-Müller, M., and Hirsekorn, S., "Atomic force acoustic microscopy," in [Acoustic Scanning Probe Microscopy], 123-153, Springer Berlin Heidelberg (oct 2012).

[13] https://en.wikipedia.org/wiki/Mathematical_morphology\#Grayscale_morphology. 